\title{
Application of calculus equation in solving thermal decomposition kinetics parameters of flame retardant flexible polyurethane foam
}

\author{
Caiyun SUN ${ }^{1, a}$, Genyin CHENG ${ }^{2, b}$, Ming GAO ${ }^{3, c}$ \\ ${ }^{1}$ Department of Foundation, North China University of Science and Technology, Box 206, Yanjiao \\ Beijing 101601, China \\ ${ }^{2}$ Occupation Safety and Health Cooperative Innovation Center, North China University of Science \\ and Technology, Box 206, Yanjiao Beijing 101601, China \\ ${ }^{3}$ School of Environmental Engineering, North China University of Science and Technology, Box \\ 206, Yanjiao Beijing 101601, China \\ a94178480@ncist.edu.cn, b1298275966@qq.com, cgaoming@ncist.edu.cn
}

Keywords: Broido equation, activation energy, pyrolysis, flexible polyurethane foam.

\begin{abstract}
Flexible polyurethane foam has been treated with borax, expanded graphite (EG), EG/borax as flame retardant, respectively. The thermal degradation of samples has been studied by thermogravimetry (TG) and differential thermogravimetry (DTG) in air. From the resulting data, kinetic parameters for different stages of thermal degradation are obtained following the method of Broido. For the decomposition of flexible polyurethane foam and flame retardant flexible polyurethane foam, the activation energy is found to increase from 21.8 to $37.6 \mathrm{kJmol}^{-1}$, which shows the good flame retardance of these compounds.
\end{abstract}

\section{Introduction}

Flexible polyurethane foam (FPUF) is widely used as furniture mat, seat cushion, various kinds of soft liner of laminated composite materials, as filtering materials, insulation materials, shock-proof materials, decoration materials, packaging materials and heat insulation materials, etc. The use of flexible polyurethane foam in house interiors, building or public transport constitutes a potential hazard for people in case of fire [1-2]. The need for consumer protection, coupled with the new regulations and environmental concerns, increases the interest in flame-retardant treatments. Halogenated flame retardants are generally used in most engineering plastics because of their excellent retardant performance [3]. However, the severe environmental impact of the processing and combustion of various brominated flame retardants motivated the investigation into halogen-free flame retardants to replace brominated and chlorinated ones [4]. Graphite and metallic hydroxide flame retardants are the most interesting and promising halogen-free flame retardants [5]. Expanded graphite is widely used to increase char yield and promote the thermal stability of intumescent flame retardants (IFR) because of their low cost and simple preparation with most polymers [6]. Generally, the presence of graphite leads to the formation of a protective thermally stable surface layer, which limits the heat transfer from the flame to the substrate and mass transfer from the substrate to the flame. The overall rate of flame feeding by combustible products from polymer pyrolysis and thermo-oxidation is therefore decreased. Because borax has certain flame retardant effect, take away a lot of heat and water loss when heating, water loss after the powder will be covered on the surface of material, which will make it difficult to burn [7].

In the present study, FPUF was treated with borax, expanded graphite (EG), EG/borax as flame retardant, respectively. The thermal degradation of samples has been studied by TG, the values of different kinetic parameters of flexible polyurethane foam and modified flexible polyurethane foam were calculated by the Broido equation [8], providing a theoretical basis for the study of the fire retardant mechanism. 


\section{Experimental}

\section{Chemicals.}

Borax $\left(\mathrm{Na}_{2} \mathrm{~B}_{4} \mathrm{O}_{7} \cdot 10 \mathrm{H}_{2} \mathrm{O}\right.$; analytical reagent) was supplied by Tianjin Yongda Chemical Reagent Co. EG (ADT150) was supplied by Shijiazhuang Ke Peng flame retardant material factory. Polyether polyols mixture (Cst-1076A/B) and isocyanurate (MDI; Cst-1076A/B) was supplied by Shenzhen Kesheng Trading Company ltd.

\section{Sample preparation.}

Isocyanate, polyether polyols, and dimethyl silicone oil of were well mixed in a $1 \mathrm{~L}$ beaker. Next flame retardant was added into the beaker with vigorous stirring for $10 \mathrm{~s}$. FPUF was treated with borax, EG, $4 \mathrm{~g}$ EG/2 g borax, respectively. The mixture was immediately poured into an open mold $\left(300 \times 250 \times 150 \mathrm{~mm}^{3}\right)$ to produce free-rise foam. Foam blocks so obtained were kept in an oven at $70^{\circ} \mathrm{C}$ for $24 \mathrm{~h}$ to complete the polymerization reaction. Samples were cut into the desired shape and size by rubbing with fine emery paper, and these test species were used for the evaluation of different properties.

\section{Thermal analysis.}

Thermogravimetry (TG) and differential thermogravimetry (DTG) were carried out on a DT-40 thermal analyzer (Shimadzu, Japan). DTA and TG were performed under a dynamic air (dried) atmosphere at a heating rate of $10^{\circ} \mathrm{Cmin}^{-1}$.

\section{Results and discussion.}

The simultaneous DTG and TG curves of samples (1-4) were carried out in a dynamic air from ambient temperature to $900^{\circ} \mathrm{C}$ and are shown in Figures 1-4. The weight losses for various stages in TG curves are given in Table 1.
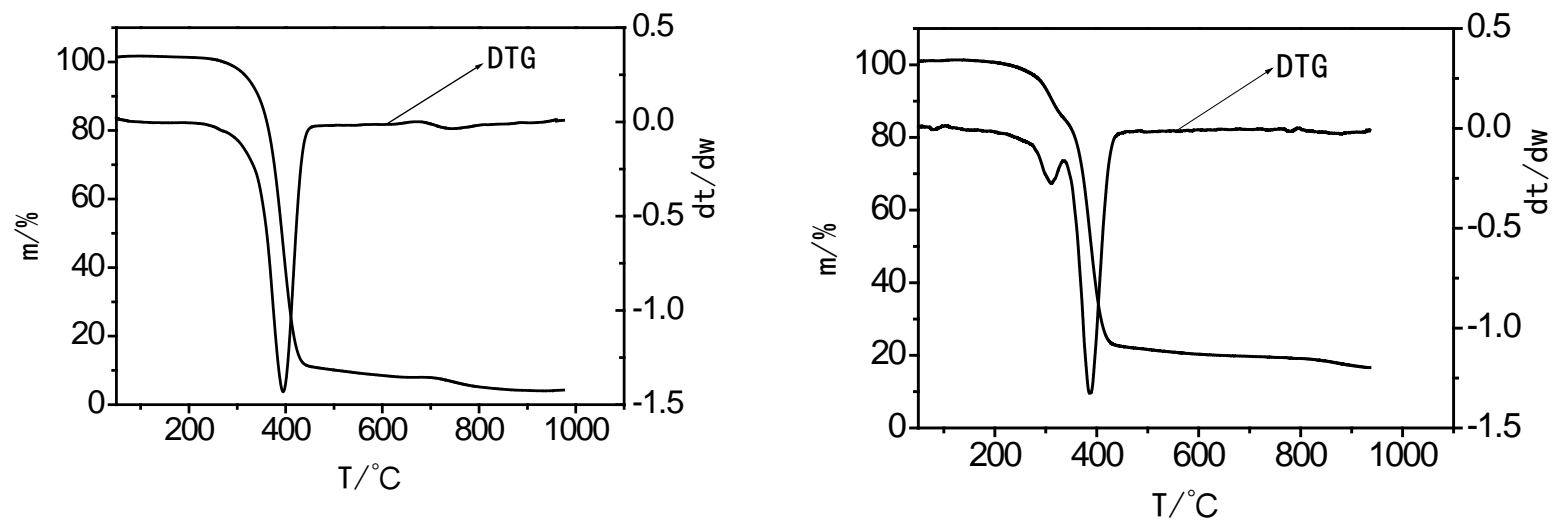

Fig.1 TG curves of sample 1 in air. Fig.2 TG curves of sample 1 in air.
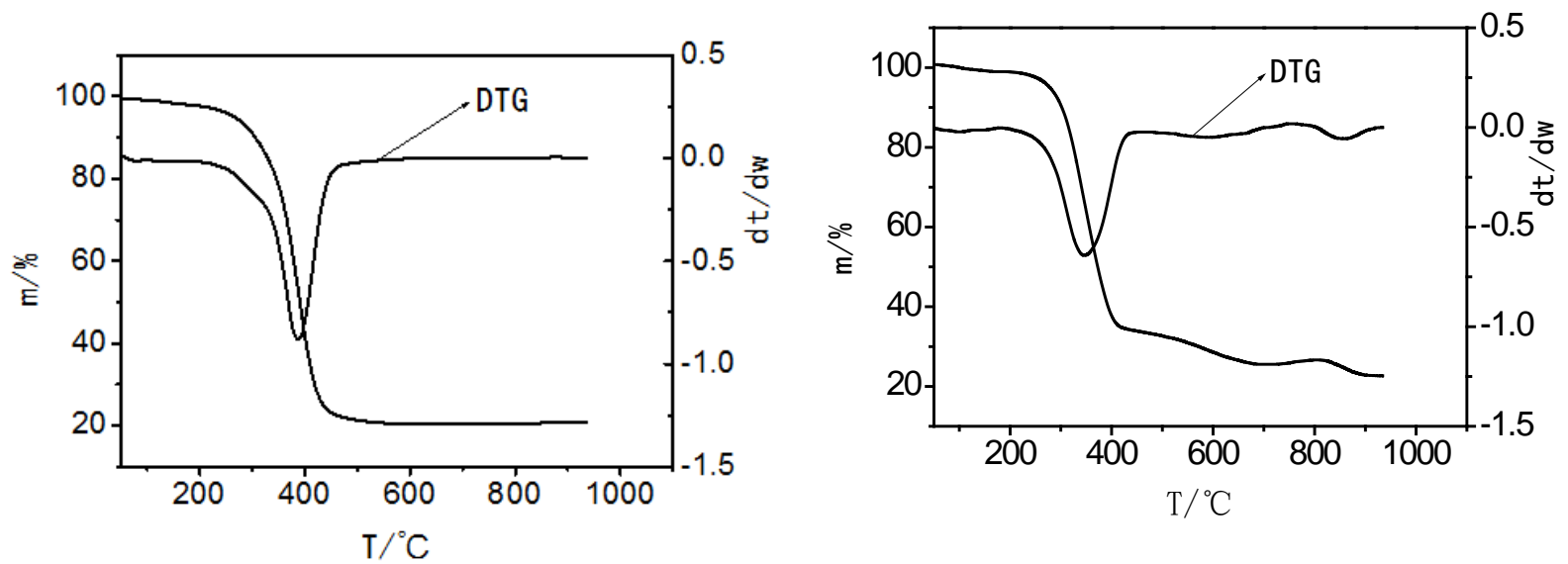

Fig.3 TG curves of sample 1 in air. Fig.4 TG curves of sample 1 in air.

TG curves of flexible polyurethane foam samples show three stages of the thermal degradation. There is a weight loss in the temperature range $20-300^{\circ} \mathrm{C}$ in the first stage, which is due to 
dehydration of flexible polyurethane foam. For the second stage, rapid weight loss occurs in the temperature range $300-400^{\circ} \mathrm{C}$, which is due to the oxidative decomposition of flexible polyurethane foam. In this stage, the less stable aliphatic groups are preferentially decomposed through hemolytic cleavage of C-C and C-H bonds, and the resultant product is a highly condensed and cross linked carbonaceous which is a little more stable. After the oxidative decomposition stage, weight losses of the residual materials of flexible polyurethane foam are found to be slow in the temperature range $400-900^{\circ} \mathrm{C}$, which is due to the oxidation of the carbonaceous.

For the second stage of thermal degradation of flexible polyurethane foam, the kinetic parameters for these stages were determined using the following equation, given by Broido [8]:

$$
\ln \left(\ln \frac{1}{\mathrm{y}}\right)=-\frac{\mathrm{Ea}}{\mathrm{R}} \cdot \frac{1}{\mathrm{~T}}+\ln \left(\frac{\mathrm{R}}{\mathrm{Ea}} \cdot \frac{\mathrm{Z}}{\beta} \cdot \mathrm{T}_{\mathrm{m}}^{2}\right)
$$

Where $y$ is the fraction of initial molecules not yet decomposed. $T_{m}$ is the temperature of maximum reaction rate, $\beta$ is the rate of heating, $\mathrm{Z}$ is the frequency factor, and Ea is the activation energy.
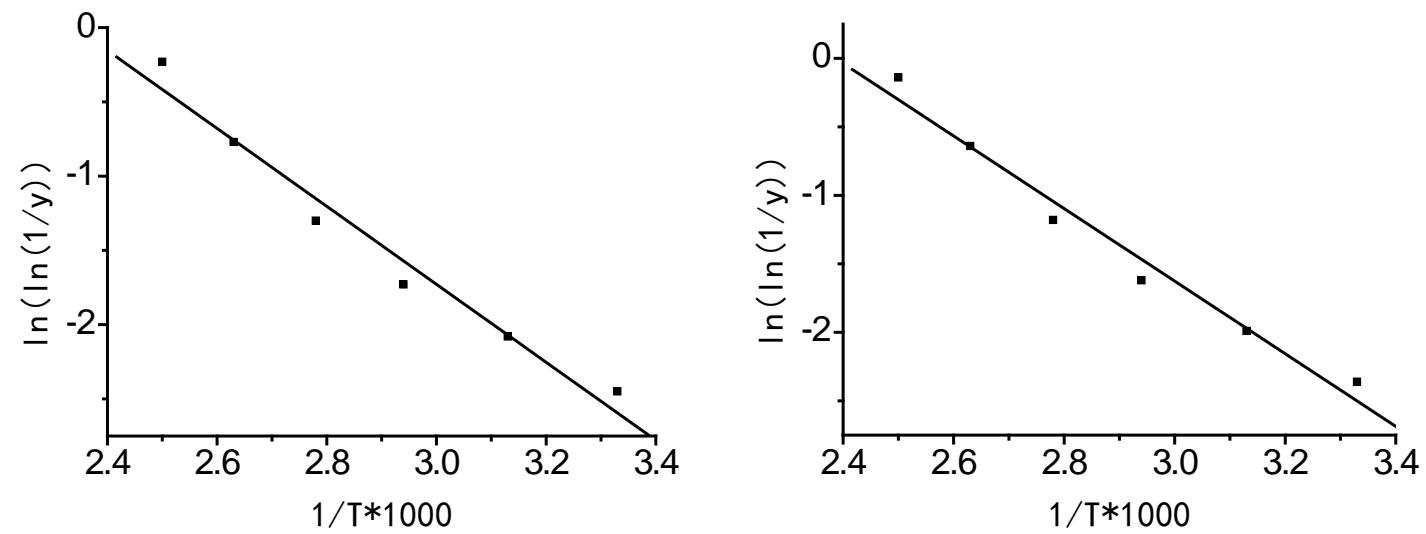

Fig.5 Plots of $\ln [\ln (1 / y)]$ versus $10^{3} / \mathrm{T}$ using Broido equation for sample 1(Left) and 2 (Right)
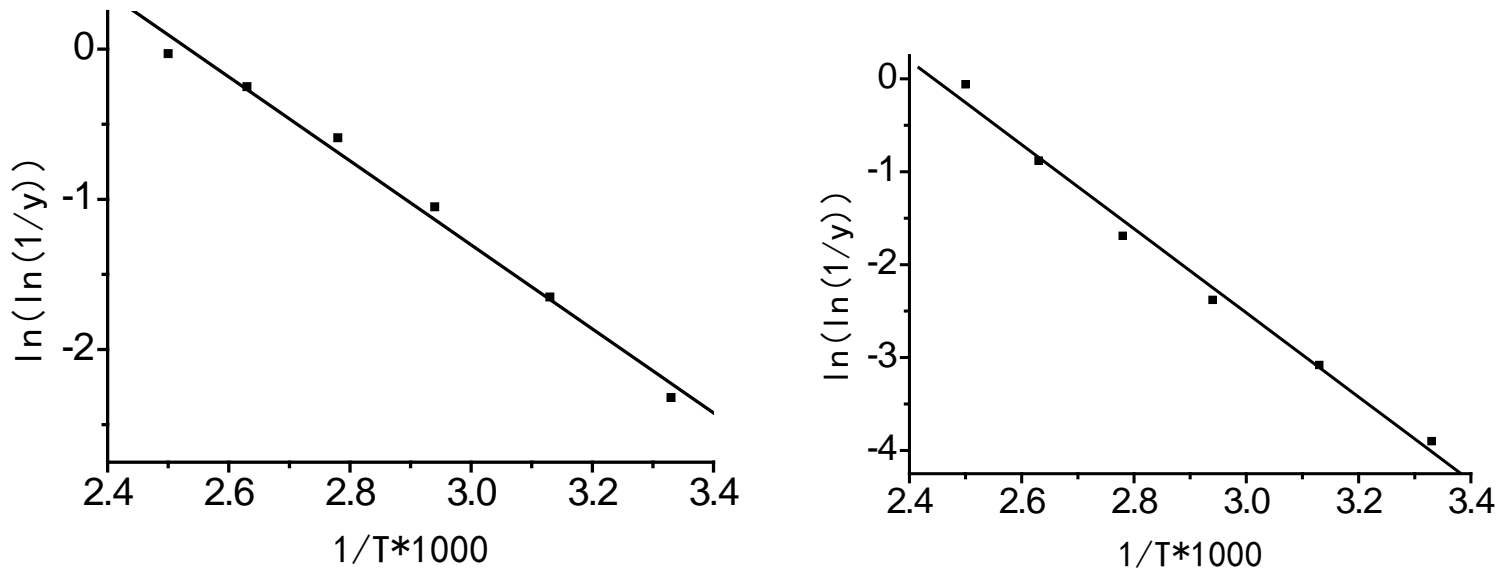

Fig.6 Plots of $\ln [\ln (1 / y)]$ versus $10^{3} /$ T using Broido equation for sample 3 (Left) and 4 (Right)

From the slopes of the TG curves in Fig 1, plots of $\ln (\ln 1 / y)$ versus $1 / \mathrm{T}$ for various stages of pyrolysis were drawn. Linear plots were obtained in each instance, and are shown in Figs 5-6. The activation energies, Ea, and the correlation coefficient, R, determined from the slopes and intercepts, respectively, of these plots are given in Table 1. From Table 1, it is observed that for the second stage of thermal degradation of flexible polyurethane foam, mainly due to dehydration, the activation energy is $21.8 \mathrm{kJmol}^{-1}$; which for samples (2-4) increase to 22.0, 23.2 and $37.6 \mathrm{kJmol}^{-1}$, respectively. This is mainly due to the increase of the thermal stability.

Generally, at lower temperatures, the degradation of flexible polyurethane foam involves dehydration, rearrangement and ultimate formation of carbonaceous residue and small amount of 
flammable products. At higher temperatures, the degradation of flexible polyurethane foam includes a rapid volatilization via the formation of a tarry mixture which ultimately decomposes into flammable volatiles and leaves small amount of char. For samples (2-4), the oxidative decomposition (second) stages occur at higher temperatures, which makes pyrolytic degradation of samples proceed difficult. This factor will give a lower percent of flammable volatile products and correspondingly higher amount of char yield.

Table 1. The kinetic parameters of samples.

\begin{tabular}{cccccccc}
\hline No. & FPUF/ml & borax /g & EG/g & Temperatures & $\mathrm{Ea} / \mathrm{KJmol}^{-1}$ & Char yield & $\mathrm{R}$ \\
\hline 1 & 20 & & & $300-405$ & 21.8 & 2.5 & -0.98489 \\
2 & 20 & 6 & & $300-415$ & 22.0 & 18.0 & -0.98729 \\
3 & 20 & & 6 & $300-406$ & 23.2 & 19.0 & -0.99451 \\
6 & 20 & 2 & 4 & $300-407$ & 37.6 & 22.0 & -0.99508 \\
\hline
\end{tabular}

In the case of samples (2-4), the lowering of the decomposition temperatures is much great. Their activation energies for the second stage of pyrolysis, in a range of 22-37.6 kJmol ${ }^{-1}$, increase considerably as compared to flexible polyurethane foam. This is due to inhibit decomposition of flame retardant. This shows that the flame-retardants change the mechanism of thermal degradation in such a way as to increase the decomposition temperatures of flexible polyurethane foam, which can lead to less flammable volatile products and more char to get good flame retardancy.

\section{Summary and conclusions}

For the decomposition of flexible polyurethane foam and flame retardant flexible polyurethane foam, the activation energy is found to increase from 21.8 to $37.6 \mathrm{kJmol}^{-1}$, the char yield is found to increase from 2.5 to $22.0 \%$, which indicates that the flame retardants inhibit decomposition of flexible polyurethane foam, which makes the whole oxidative decomposition of flexible polyurethane foam occur at higher temperatures, resulting in formation of less flammable products and correspondingly more char. So the flexible polyurethane foam treated with EG/borax has high flame retardant efficiency.

\section{Acknowledgments}

The work was supported by key project of North China Institute of Science and Technology (HKXJZD201402) and joint fund of the national Natural Foundation (U1361130).

\section{References}

[1] Liang, Q. Y. \& Chang, H. X. Flexible polyurethane foam flame retardant processing, Fire Science and Technology. 1995(03), 38-39.

[2] Liang, D. S, Zhou, H. L, Chen, R. H \& You, T. Q. Flame retardant technology reseach of flexible polyurethane foam, Technique and Products information. 1997(12), 18-21.

[3] Wu X, Wang L, Wu C, Yu J, Xie L, Wang G, Jiang P, Influence of char residues on flammability of EVA/EG, EVA/NG and EVA/GO composites. Polym Degrad Stab, 2012 (97),54-63.

[4] Ramani A, Dahoe AE, On the performance and mechanism of brominated and halogen free flame retardants in formulations of glass fibre reinforced poly(butylene terephthalate). Polym Degrad Stab (2014)104:71-86.

[5] Li Z, Qu B, Flammability characterization and synergistic effects of expandable graphite with magnesium hydroxide in halogen-free-retardant EVA blends. Polym Degrad Stab (2003) 81:401-408. 
[6] Bai G, Guo C, Li L, Synergistic effect of intumescent flame retardant and expandable graphite on mechanical and flame-retardant properties of wood flour-polypropylene composites. Constr Build Mater (2014)50:148-153.

[7] Heng, Z. Boron-containing flame retardance, Shanghai Plastics. 2012(03), 12-18.

[8] Broido, A., A Simple, Sensitive Graphical Method of Treating Thermogravimetric Analysis Data, J. Polym. Sci. Part A-2, (1969) 7, 1761. 